\title{
Response to Investigation of an appropriate contrast-enhanced CT protocol for young patients following the Fontan operation
}

\author{
M. Kardos ${ }^{1}$
}

Received: 21 May 2018 / Accepted: 8 June 2018 / Published online: 15 June 2018

(c) Japan Radiological Society 2018

To the Editor,

I am writing in regard to the article titled "Investigation of an appropriate contrast-enhanced CT protocol for young patients following the Fontan operation" published in March 2018 issue of JJR [1].

I believe that follow-up of patients after Fontan procedure with CT angiography should be very cautious due to ionizing radiation. It should be reserved just for cases when MRI follow-up is contraindicated, including patients with pacemakers or defibrillators and those with surgical material such as hemostatic clips, stents, and embolization coils with significant susceptibility artifacts.

The authors of this paper tried to develop an adequate contrast-enhanced CT protocol for children with congenital heart diseases following the Fontan operation. The values of Hounsfield unites (HU) measured in conduits were $185 \pm 46$ in $60 \mathrm{~s}$ protocol and $185 \pm 31 \mathrm{HU}$ in $70 \mathrm{~s}$ protocol, respectively. This mean value of $\mathrm{HU}$ is sufficient for the evaluation of Fontan pathway, but its applicability to older patients is in question. Because the authors evaluate just patients aged 2-11 years (median 5 years). I do not think that this protocol with 60s and 70s delayed scan is applicable for older patients. In my practice, I try to reach these values of HU with using dual bolus injection technique with single acquisition. This technique was first time performed by Thomas in pediatric multi-region body CT, but it is well applicable to the imaging follow-up after Fontan procedure [2].
Our CT imaging protocol for Fontan patients includes a weight-stratified dual bolus intravenous contrast technique with single acquisition. In this way the authors can achieve $\mathrm{HU}$ values similar to their protocol in older patients.

Sincerely,

Funding The author(s) received no financial support for the research, authorship, and/or publication of this article.

\section{Compliance with ethical standards}

Conflict of interest The author(s) declared no potential conflicts of interest with respect to the research, authorship, and/or publication of this article.

Ethical statement All applicable institutional and/or national guidelines for the care and use of animals were followed.

\section{References}

1. Nakagawa M, Ozawa Y, Nomura N, Inukai S, Shiba A, Sakurai $\mathrm{K}$, Shimohira M, Shibamoto Y. Investigation of an appropriate contrast-enhanced CT protocol for young patients following the Fontan operation. Jpn J Radiol. 2018;36:215-22. https://doi. org/10.1007/s11604-018-0718-3.

2. Thomas KE, Mann EH, Padfield N, et al. Dual bolus intravenous contrast injection technique for multiregion paediatric body CT. Eur Radiol. 2015;25:1014-22. https://doi.org/10.1007/s0033 0-014-3501-6.
M. Kardos

kardi.marek@gmail.com

1 Department of Functional Diagnostics, Children's Cardiac Center, Limbova 1, 83351 Bratislava, Slovakia 